\title{
MINIMAL LINE GRAPHS
}

\author{
by DAVID P. SUMNER
}

(Received 1 October, 1974; revised 18 March, 1975)

In this paper all graphs will be ordinary graphs, i.e. finite, undirected, and without loops or multiple edges. For points $x$ and $y$ of a graph $G$, we shall indicate that $x$ is adjacent to $y$ by writing $x \perp y$, and if $x$ is not adjacent to $y$ we shall write $x \pm y . \dagger$ We shall denote the degree of a point $x$ by $\delta(x)$ and the minimal degree of $G$ by $\delta(G)$.

By the line graph of a graph $G$ we shall mean the graph $L(G)$ whose points are the edges of $G$, with two points of $L(G)$ adjacent whenever they are adjacent in $G$. A graph $G$ is said to be a line graph if there exists a graph $H$ such that $G=L(H)$.

Line graphs have been characterized in various ways. Two of these are given in Lemmas 1 and 2. Beineke [1] has determined a collection of nine graphs with the property that a graph is a line graph exactly when it does not have one of these graphs as an induced subgraph. Proofs of all three of these characterizations may be found in Harary [2].

Definitions. (1) By an $E C$-partition for the graph $G$ we mean a partition $E$ of the edges of $G$ satisfying

(i) each element of $E$ induces a complete subgraph of $G$, and

(ii) no point of $G$ lies in more than two of the graphs induced by the elements of $E$.

If $A \in E$, then $\langle A\rangle$ will denote the subgraph induced by $A$. Thus the points of $\langle A\rangle$ consist of those points of $G$ which are incident with some element of $A$ and the edges of $\langle A\rangle$ consist of the elements of $A$.

(2) A triangle in $G$ is odd if there is a point adjacent to an odd number of its vertices. A triangle which is not odd is said to be even.

(3) By a terminal $n$-clique $E$ of $G$ we mean a clique of order $n$ which is either equal to $G$ or has exactly one point of $E$ adjacent to points outside $E$ (a clique is a maximal complete subgraph).

(4) If $e$ is an edge of $G$, we shall denote the graph obtained from $G$ by deleting $e$ by $G-e$.

LEMmA 1 (Krausz [3]). A graph $G$ is a line graph if and only if $G$ has an EC-partition.

Lemma 2 (van Rooij and Wilf [4]). A graph $G$ is a line graph if and only if (1) $G$ does not have $K_{1,3}$ as an induced subgraph and (2) if two odd triangles in $G$ share a common edge, then their points induce a complete graph on four points.

It is obvious that every induced subgraph of a line graph is again a line graph. Thus the graph obtained from a line graph by deleting a single point is also a line graph. However the situation is different with respect to edge deletion, since an arbitrary subgraph of a line graph

† This neat notation was taken from D. J. Foulis. 
need not be again one such. In fact, there exist line graphs $G$ such that, for every edge $e, G-e$ is not a line graph. We shall call such a graph a minimal line graph. It is easy to see that any complete graph of order at least five is a minimal line graph.

Our purpose in this paper is to characterize line graphs which are minimal. For this purpose it is, of course, only necessary to characterize connected minimal line graphs.

REMARK. It is known that the only connected line graphs which contain two even triangles sharing a common edge are the three graphs of Figure 1 (see Harary [2], p. 77).

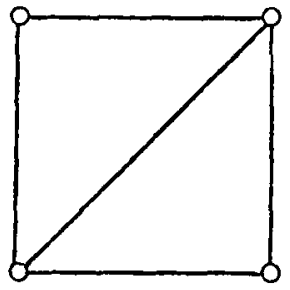

(a)

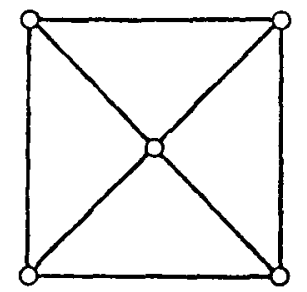

(b)

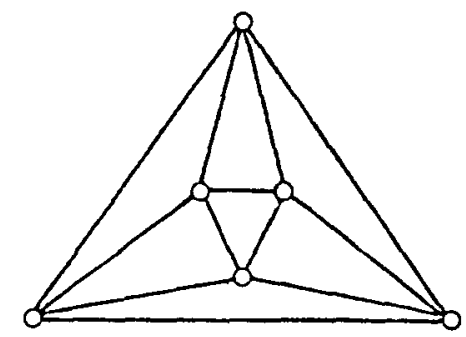

(c)

Figure 1

THeOREM 1. A connected nontrivial line graph $G$ is minimal if and only if the following conditions hold:

(1) every edge of $G$ lies in a triangle,

(2) $\delta(G) \geqq 3$,

(3) every edge of an even triangle lies in at least one other triangle, and

(4) G has no terminal 4-cliques.

Proof. We first show that the conditions (1)-(4) are necessary. Let $G$ be a connected, nontrivial minimal line graph.

(1) Suppose that there is an edge $e$ of $G$ which does not lie in any triangle. Let $E$ be an EC-partition for $G$. Since $e$ lies in no triangle, $\{e\} \in E$. Hence $E_{0}=E-\{\{e\}\}$ is an ECpartition for $G-e$ and so $G-e$ is a line graph by Lemma 1. Thus (1) holds.

(2) Since every edge of $G$ lies in a triangle, $\delta(G) \geqq 2$. Suppose there exists $x \in G$ with $\delta(x)=2$, and let $a$ and $b$ denote the points adjacent to $x$. The edge $a x$ must lie in a triangle and so we must have $a \perp b$. Let $e=a x, f=a b$ and $g=b x$, and let $E$ be an EC-partition for $G$. Then if $\{e\} \in E$, we would have that $E_{0}=E-\{\{e\}\}$ is an EC-partition for $G-e$. If $\{e\} \notin E$, then since the only other complete subgraph containing $e$ is the triangle $a b x,\{e, f, g\} \in E$. But in this case we may form

$$
E_{1}=(E-\{\{e, f, g\}\}) \cup\{\{e\},\{g\}\}
$$

which satisfies the conditions to be an EC-partition for $G-f$. (Note that since $\delta(x)=2, x$ lies only in the graphs induced by $\{e\}$ and $\{g\}$.) Thus $G-f$ is a line graph and (2) holds.

(3) We note that the only minimal line graph which contains two even triangles sharing a 
common edge is the graph of Figure 1(c). Since condition (3) holds for this graph, we shall assume that $G$ does not possess two even triangles with an edge in common. Let $T=a b x$ be an even triangle in $G$, and suppose that $e=a b$ does not lie in any other triangle in $G$. Then since $a$ and $b$ both have degree at least three and $T$ is even, there exist $w, z \in G-T$ with $w \perp a, w \perp x$, $w \pm b, z \perp b, z \perp x$ and $z \pm a$. Let $E$ be an EC-partition for $G$. We claim that $\{e\} \in E$; for suppose that $\{e\} \notin E$. Then since $T$ is the only other complete subgraph of $G$ containing $e$, $A=\{a b, a x, b x\} \in E$. Thus $x \in\langle A\rangle$ and hence, since $x$ can only lie in two of the subgraphs induced by the elements of $E, w x$ and $x z$ must lie in the same element of $E$ and so $w \perp z$. But since $G$ has no two even triangles sharing an edge, wax and $z x b$ are odd triangles. Moreover, $w \pm b$ so that by Lemma $2, w x z$ is an even triangle. Thus $w x z$ is a clique and hence $B=\{w x, x z, w z\} \in E$. Therefore, every edge incident with $x$ lies in $A \cup B$ and hence no point not in $H=\{a, b, x, w, z\}$ is adjacent to $x$. Also since $T$ is even, any point in $G-H$ which is adjacent to $a$ must be adjacent to $x$. Thus we see that $a$ is adjacent to no point in $G-H$. But since wax is odd, there exists $t \in G-H$ with $t \perp w$ and $t \pm a$. But then $w$ lies in $\langle B\rangle$ as well as the subgraphs induced by the elements of $E$ containing $a w$ and $w t$. But this is impossible, so that $\{e\} \in E$. Thus $E_{0}=E-\{\{e\}\}$ is an EC-partition for $G-e$ and so $G-e$ is a line graph. Hence (3) holds.

(4) Suppose that $G$ contained the terminal 4-clique $F=\{a, b, c, d\}$. Since $F$ is not itself a minimal line graph, we may suppose that $d$ is the unique point of $F$ adjacent to some point $x \notin F$. In this case if $E$ is an EC-partition for $G$, then, since $x$ is not adjacent to any vertex of $F$ other than $d$, we find that $a d, b d$ and $c d$ must all lie in the same element $R \in E$. Thus since $\langle R\rangle$ is complete, $R$ consists of all the edges of $F$. Therefore

$$
E_{0}=(E-\{R\}) \cup\{\{a d, d c, a c\},\{b a\},\{b c\}\}
$$

is an EC-partition for the graph obtained by deleting the edge $b d$. Thus (4) holds.

Now suppose that $G$ is a connected nontrivial line graph which satisfies the conditions (1)-(4). We shall show that $G$ is a minimal line graph. Let $e=x y$ be an edge of $G$. If $e$ lies in a complete subgraph of order five or more, then the deletion of $e$ will result in two odd triangles sharing an edge but not spanning a complete graph of order four. We shall therefore assume that $e$ lies in no complete subgraph of order five.

Suppose that $e$ lies in the complete subgraph $A=\{x, y, a, b\}$ of order four. We consider cases.

Case 1. Neither a nor $b$ is adjacent to points outside $A$. Then since $A$ cannot be a terminal 4-clique, both $x$ and $y$ must be adjacent to points outside $A$ and thus in $G-e$, the triangles $x a b$ and $y a b$ are odd and share an edge but do not span a complete graph of order four.

Thus $G-e$ is not a line graph, by Lemma 2 .

Case 2. There exists $z \in G-A$ such that $z \perp a$ or $z \perp b$. Without loss of generality suppose that $z \perp a$. If $z \pm x$ and $z \pm y$, then $\{a, x, y, z\}$ would induce a $K_{1,3}$ in $G-e$. Thus we may suppose that $z \perp x$. We note that $z$ is not adjacent to both $b$ and $y$ since $e$ does not lie in any complete subgraph of order five. However, if $z$ is adjacent to exactly one of $b$ and $y$, then $A \cup\{z\}$ induces a complete subgraph of order five minus one edge, which is impossible in a line graph. Thus $z \pm b$ and $z \pm y$. But now $x a b$ is an odd triangle sharing an edge with $z x a$ and 
so, since $z \nmid b$, we see that zax must be an even triangle. But then the edge $z a$ must lie in another triangle, so that there exists $w \in G-\{x\}$ with $w \perp z$ and $w \perp a$. We may assume that the triangle $w z a$ is odd since the theorem is easily checked for the three line graphs having two even triangles which share a common edge. However, in this case we see that the triangles wza and $z x a$ are odd triangles in $G-e$ and $w \neq x$ since $z a x$ is even in $G$.

Therefore in this case also, $G-e$ is not a line graph.

Hence we may suppose that $e$ does not lie in any complete graph of order four. Let $T=c x y$ be a triangle containing $e$.

Suppose that $T$ is even. Since $e$ must lie in a triangle other than $T$, there exists $r \in G-\{c\}$ with $r \perp x, r \perp y$ and $r \pm c$. Also since $\delta(r) \geqq 3$, there exists $u \in G-\{x, y\}$ with $u \perp r$. If $u \pm x$ and $u \pm y$, then $\{r, u, x, y\}$ would induce $K_{1,3}$ in $G-e$. Hence we may suppose that $u \perp y$. Since $e$ does not lie in any complete subgraph of order four, $u \pm x$. Thus by the evenness of $T$, we have $u \perp c$. But then ruy and cuy are odd triangles in $G-e$ with $r \pm c$.

Finally, suppose that $T$ is odd. Since $\delta(c) \geqq 3$, we may find $z \in G-\{x, y\}$ with $z \perp c$. In order that $\{z, c, x, y\}$ does not induce $K_{1,3}$ in $G-e$, we may assume that $z \perp x$. However, $e$ lies in no complete subgraph of order four, so that $z \neq y$. Moreover, since $T$ is odd and $y \neq z$, it must be that $x c z$ is an even triangle. Hence $z c$ lies in a triangle other than $x c z$, so there exists $w \in G-\{x\}$ with $w \perp z$ and $w \perp c$. But, as before, we may suppose that no two even triangles in $G$ share a common edge and thus $w c z$ is odd in $G$. Hence in $G-e, y$ is adjacent to exactly one point of $w c z$ and hence $x c z$ and $w c z$ are odd triangles in $G-e$ with $x \pm w$.

Hence once again by Lemma $2, G-e$ is not a line graph.

COROLLARY 1. A connected, nontrivial line graph $G$ which is not the graph in Figure 1(c) (i.e. $G$ is not the line graph of $K_{4}$ - the complete graph on four points) is a minimal line graph if and only if

(1) Every edge of $G$ lies in an odd triangle,

(2) $\delta(G) \geqq 3$, and

(3) G has no terminal 4-cliques.

For $x$ a point of $G$, we shall let $x^{\perp}$ denote the set of all points of $G$ adjacent to $x$.

COROLlaRY 2. If $x$ is a point of minimal line graph $G$ with $\delta(x)=3$, then $\{x\} \cup x^{\perp}$ is complete.

Proof. Let $x^{\perp}=\{a, b, c\}$. Then since every edge of $G$ lies in a triangle, we may suppose without loss of generality that $a \perp b$ and $b \perp c$. If $a \pm c$, then, by Lemma 2, one of $a b x$ and $b c x$ is even and so one of the edges $a x$ and $c x$ lies in two triangles, which is impossible if $a \pm c$.

Since a complete graph of order four is not a minimal line graph, we have

COROLlaRY 3. There are no cubic minimal line graphs.

Our next theorem characterizes those connected graphs whose line graphs are minimal.

THEOREM 2. Let $H$ be a connected graph with at least three points. Then $L(H)$ is a minimal line graph if and only if $H$ satisfies the following three conditions: 
(1) H has no points of degree two,

(2) If $x$ is a point of $H$ which is adjacent to an endpoint, then $\delta(x) \geqq 4$, and

(3) If $x$ is a point of $H$ which is adjacent to three or more endpoints, then $\delta(x) \geqq 5$.

Proof. Since the theorem is easily checked for graphs with four or fewer points, we may assume that $H$ has at least five points.

Let $G=L(H)$. Then since $|H| \geqq 5$ and $H$ is connected, the condition that $H$ has no points of degree two is equivalent to the requirement that every edge of $G$ lies in an odd triangle. Condition (3) above is equivalent to the condition that $G$ does not contain any terminal 4-cliques. Conditions (1) and (2) imply that $\delta(G) \geqq 3$, and if $\delta(G) \geqq 3$, then $H$ satisfies (2). Hence since $|H| \geqq 5, G$ cannot be the line graph of $K_{4}$ and thus the theorem follows at once from Corollary 1.

COROLLARY 4. If $H$ is a connected graph with $\delta(H) \geqq 3$, then $L(H)$ is a minimal line graph.

\section{REFERENCES}

1. L. W. Beineke, Characterizations of derived graphs, J. Comb. Theory 9 (1970), 129-135.

2. F. Harary, Graph Theory (Reading, Massachusetts: Addison-Wesley Publishing Company, 1969).

3. J. Krauz, Démonstration nouvelle d'un théorème de Whitney sur les réseaux, Mat. Fiz. Lapok 50 (1943), 75-89.

4. A. van Rooij and $\mathrm{H}$. Wilf, The interchange graphs of a finite graph, Acta Math. Acad. Sci. Hungar. 16 (1965), 263-269.

University of South Carolina

COLUMBia

South Carolina 29208, U.S.A. 